\title{
Development and engineering of artificial RNAs
}

Editor: Michael RYCKELYNCK

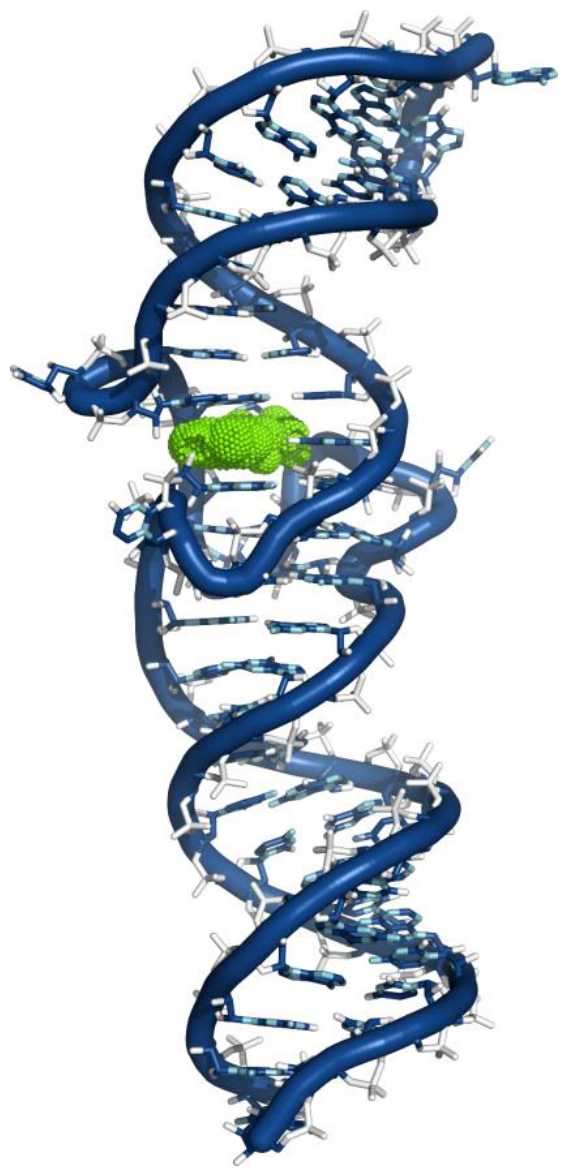

Cover legend: Crystal structure of the artificial light-up RNA aptamer iSpinach in complex with its fluorogen. The aptamer is shown in blue and the co-factor in green (PDB 5OB3). 


\section{Running order:}

1. Künne et al.

2. Boussebayle et al.

3. Wang et al.

4. Karunanayake Mudiyanselage et al.

5. Sack et al.

6. Yokobayshi

7. Autour et al.

8. Hammer et al.

9. Flamme et al.

10. Randrianjatovo-Gbalou and Delarue

\section{Sections Headings:}

1. New de novo selection methods of artificial RNA (papers 1 and 2)

2. Engineering of artificial RNA (papers 3 to 8 )

3. Synthesis of non-natural RNA derivatives (papers 9 and 10) 


\section{Editorial}

In 1957, Francis Crick enounced the central dogma of molecular biology according to which the genetic information stored in the DNA flows toward its translation into proteins via the use of labile messenger RNA (mRNA) intermediates [1]. Nowadays, this central role of RNA in cell life has become even more obvious when considering the vast variety of non-coding RNAs at work in the cell. These RNAs are endowed with key roles not only in gene expression, by directly participating in mRNA translation (e.g., ribosomal and transfer RNAs), but also by being key effectors of its regulation. Indeed, non-coding regulatory RNAs have now been uncovered in every living system (from bacteria to mammals) and even in viruses [2,3]. These RNAs can act at several levels all along the mRNA lifespan and may, for instance, activate or repress mRNA synthesis, regulate mRNA translation (as is the case with, e.g., small non-coding RNAs in bacteria and microRNAs in mammals) or even modulate mRNA stability. Besides non-coding RNAs acting in trans, regulatory function can also be supported by a specialized domain embedded within the mRNA itself. This is typically the case for riboswitches, which are structure-switching elements mainly found in bacteria that can adopt alternative folds depending on the presence of a specific cofactor (usually a small metabolite or an amino acid) [4]. These RNAs are usually composed of three structural elements. First, an aptamer module interacts very specifically with the target cofactor. This interaction leads to a structural switch of the aptamer that is then transmitted by a communication module to the regulatory platform. This last element will then switch its conformation to generate (or destabilize) a transcription termination stem-loop or trap (or release) the ribosome binding site that controls transcription and translation, respectively. Beside these functions that are mainly driven by base paring and structural remodeling, a last class of RNAs termed ribozymes (standing for ribonucleic acid enzymes [5]) was discovered in the early 80's [6, 7] as RNAs able to catalyze chemical reactions such as transpeptidation, RNA cleavage or splicing. These pleiotropic functions of RNA are made possible by the great structural plasticity of a molecule composed of a small 4-letter alphabet that can rapidly evolve and acquire new functions by simple point mutations or more extensive sequence variations. This capacity of RNA to fulfill such a variety of functions together with the resulting wide application range strongly stimulated the emergence of innovative methods dedicated to the development and engineering of new artificial RNAs and their derivatives. In turn, these advances have motivated this special issue of Methods, aiming to introduce some of these emerging technologies.

Pioneered by the groups of Larry Gold and Jack Szostak [8, 9] in the early 90's, the technology known as SELEX (Systematic Evolution of Ligands by EXponential enrichment) is probably the most emblematic method dedicated to the de novo discovery of artificial RNAs. Briefly, in this method a large library (typically $10^{15}$ different sequences) of RNA molecules carrying a randomized stretch of sequence is incubated with a target prior to partitioning the bound fraction from the bulk and exponentially amplifying the recovered RNA by RT-PCR. Even though this approach is extremely efficient for the isolation of new aptamers, specifically those for targets ranging in size from metal ions to proteins, the original method suffered some limitations that are beginning to be addressed. First, when performed manually SELEX is a very time-consuming and labor-intensive process involving several rounds performed under conditions that ought to be as reproducible as possible. Yet, substantial gains in time and efficiency can be achieved by automating the process. Whereas several strategies have been attempted in the past, in this issue Künne et al. introduce the first robotic platform allowing the complete automation of the process, leading to the isolation of aptamers in only a few days in a completely unsupervised way [10]. A second significant limitation of the original SELEX arises when one is looking at isolating structure-switching aptamers for the design of artificial riboswitches or biosensors (see below). Indeed, conventional SELEX allows isolating aptamers able to specifically recognize and strongly interact with their target, but these aptamers also tend to have a rigid structure. Yet, a variant of the original method known as capture-SELEX was later developed allowing for selecting DNA 
molecules prone to switch their structure in response to the presence of a ligand. This method was mainly used to search for DNA switching aptamers. In their present contribution, Boussebayle et al. now report on a procedure optimized for the selection of structure-switching RNA aptamers targeting small molecules [11]. Such RNAs should greatly ease the future discovery of artificial riboswitches and biosensors.

Once isolated, aptamers can be used without additional modification, yet in many cases they are being further engineered into more complex molecules. Such engineering is exemplified by light-up RNA aptamers, a class of aptamers able to interact with and activate the fluorescence of small profluorescent compounds called fluorogens. When expressed in cells, these molecules form a fluorescent complex as soon as they are synthesized, which enables inserting them directly into the sequence of the studied RNA. In this issue, Wang et al. review the main strategies available to image mRNAs in living cells prior to presenting their Aptamer initiated Fluorescence Complementation (AiFC) method that allows to image mRNAs in living cells without the need of modifying the target mRNA [12]. In addition, Karunanayake Mudiyanselage et al. review how light-up RNA aptamers can be further engineered into genetically encoded biosensors of small molecules before presenting in more details their "Catalytic Hairpin Assembly RNA circuit that is Genetically Encoded" (CHARGE) technology they recently developed for detecting low abundant molecules with high sensitivity [13]. In both aforementioned examples, RNA was engineered by rational approaches in which the experimenter chooses the positions to mutate and the identity of the nucleotides to insert. Yet, an alternative strategy consists in randomizing a region of the molecule and then functionally screening the different mutants of the library to isolate those displaying the features of interest. Screening approaches are particularly appealing for the development of modules endowed with complex functions difficult to computationally design. For instance, the communication modules found in artificial riboswitches and biosensors often are designed using a sub-optimal trial-and-error strategy. In this issue, Sack et al. circumvent this limitation using an in vivo screening strategy allowing to identify neomycin-responsive aptazymes (a self-cleaving ribozyme whose activity is controlled by a sensing aptamer) with an optimized communication module [14]. To do so, an aptazyme consisting of a hammerhead ribozyme connected to a neomycin-specific structure-switching aptamer via a 5-nucleotide long randomized region was inserted into the 5, untranslated region of a reporter gene coding for the Green Fluorescent Protein (GFP). Constructs were then introduced and expressed in yeast before selecting those cells that displayed a differential reporter expression (that is, a differential GFP fluorescence) in the presence of neomycin. When using selfcleaving ribozymes, substantial gain in throughput can be obtained by exchanging the functional screening for a more global approach in which all the ribozymes of the library are allowed to react in a single tube, and the self-cleaving rate of each sequence is assessed by Next Generation Sequencing (NGS). This NGS-based strategy has been extensively used by Yohei Yokobayashi who reviews in this issue the main features and achievements of this approach [15]. As an alternative to in vivo screening, Autour et al. present an innovative in vitro ultrahigh-throughput functional screening strategy based on the use of droplet-based microfluidics coupled with NGS analyses [16]. In this method, a structure switching theophylline responsive aptamer was connected to a destabilized form of the light-up aptamer iSpinach via two stretches of four randomized residues. The genes of the resulting library were then individualized into picoliter volume water-in-oil droplets where they were amplified, expressed and individually assayed for the capacity of the encoded RNA to generate fluorescence in a theophyllinedependent manner. Droplets were then sorted accordingly and the whole experiment analyzed by NGS. Using this approach, more than 65,000 mutants were functionally analyzed in a single experiment and an optimal theophylline sensor identified. Besides functional screening, an exciting way of engineering functional RNAs makes use of bioinformatic prediction algorithms. In this issue, Hammer et al. review the main computational methods and prediction tools currently used for de novo conception of functional RNAs, together with the next challenges these approaches will have to address [17]. 